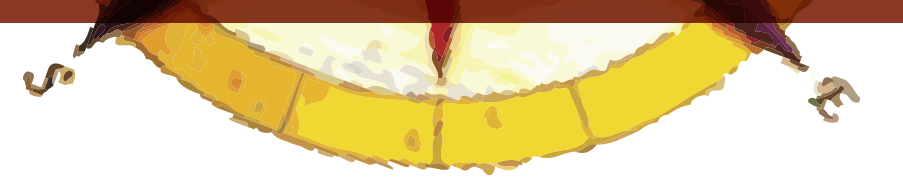

\title{
LOS TEXTOS ESCOLARES ENTRE LA ENSEÑANZA DE CONTENIDOS O EL DESARROLLO DE COMPETENCIAS
}

\author{
Mario Fernando Hurtado Beltrán ${ }^{1}$
}

\section{RESUMEN}

El presente artículo está orientado a analizar desde la política educativa de Colombia la dualidad que enfrentan los profesores entre seleccionar textos que privilegien la enseñanza de contenidos o aquellos que se enfoquen en el desarrollo de competencias y para ello es necesario saber: ¿Cómo trabajan los profesores en el aula? y isi comprenden el trabajo con competencias y habilidades?. E1 caso colombiano es una excusa o marco referente para comprender lo que pasa en América Latina y proponer una forma de desarrollar los textos escolares para la enseñanza de la geografía y la historia. El texto escolar sigue siendo una herramienta central para el trabajo del profesor en el aula, y así como cambian la orientación metodológica y didáctica de la geografía y de la historia, con el paso de los años y el surgimiento de nuevas tendencias, el texto escolar debe cambiar, pero el reto de cambiar la orientación didáctica de los libros de texto deber estar acompañada por la transformación del imaginario que tienen los profesores sobre el texto escolar.

Palabras Clave: Textos escolares, enseñanza de contenidos, desarrollo de competencias.

\section{RESUMO}

O presente artigo está orientado a analisar a partir da política educativa de Colômbia a dualidade que enfrentam os professores entre selecionar textos que privilegiem o ensino de conteúdos aqueles que se enfoquem no desenvolvimento de competências e para isso é necessário saber: ¿Como trabalham os professores na aula? e ¿se compreendem o trabalho com competências e habilidades?. O caso colombiano é uma escusa ou marco referente para compreender o que passa em América Latina y propor uma forma de desenvolver os textos escolares para o ensino da geografia e a história. O texto escolar segue sendo uma ferramenta central para o trabalho do professor na aula, e assim como cambiam a orientação metodológica e didática da geografia e da história, com o passo dos anos e o surgimento de novas tendências, o texto escolar deve cambiar, pero o reto de cambiar a orientação didática dos livros de texto deve estar acompanhado pela transformação do imaginário que tem os professores sobre o texto escolar.

Palavras Chave: Textos escolares, ensino de conteúdos, desenvolvimento de competencias.

1 Geógrafo Universidad Nacional, Magíster en Estudios Políticos, Profesor Universidad Nueva Granada. Director Editorial Ciencias Sociales Vicens Vives Colombia. 


\begin{abstract}
This article is oriented to analyze from the case of educative politic from Colombia, the duality faced by teachers in front of the decision to choose between text that grant a privilege to teaching contents, or text who focus in development of competencies. In order to do that, it is necessary to know: ¿How the teachers work in classroom? And ¿Do the teachers understand work with competencies and skills? The Colombian case is an excuse or framework to understand what is happening in Latin America and to propose a way to develop school texts for geography and history teaching. School text is a central tool for teacher's work in classroom, and because methodological and didactic orientation of geography and history have changed over the years within the emergence of new tendencies, school text must change, but the challenge of changing the text didactic orientation, should be accompanied by teachers school text imaginary transformation.
\end{abstract}

Keywords: School texts, contents teaching, competencies development.

\title{
EL DISCURSO OFICIAL
}

La enseñanza en general en la escuela y de las ciencias sociales en Colombia ha planteado un cambio (en teoría) de las formas de enseñanza a partir de las reformas educativas de 1994 y 2006. La primera en pleno auge de las políticas neoliberales, generó una reducción en el gasto estatal de la educación pública, que se orientó en el énfasis de la enseñanza del lenguaje y las matemáticas y en temáticas orientadas en la formación de valores, en detrimento de la enseñanza de la geografía y de la historia que pasaron a integrar una sola área llamada Ciencias Sociales, junto a la democracia y la política; lo que implicó una reducción de horas en la grilla de intensidad horaria semanal.. Por otra parte la reforma se centró en la evaluación por competencias, y buscó desmotivar la entrega de grandes contenidos de aprendizaje y procesos de evaluación memorística, vigentes durante gran parte del siglo XX.

Los estándares básicos de evaluación se implementaron desde 2002, cuando se realizó según el ministerio de educación una movilización nacional de expertos educativos para presentarlos a los educadores y promover su implementación. En la propuesta del MEN (Ministerio de educación nacional del Colombia) los estándares son parte de un ciclo que deben implementar las instituciones educativas llamado planes de mejoramiento, de ese modo se plantean unos estándares básicos por competencias, que deben ser evaluados y de inmediato pasar a un plan de mejoramiento para seguir fortaleciendo las competencias, es decir lo que el Ministerio propone, es que el estudiante debe saber y debe saber hacer

A pesar de las críticas, la implementación de la evaluación por competencias avanzó y ello supuso no priorizar en los contenidos y promover el desarrollo de habilidades en los estudiantes, algo que ha sido beneficioso en la educación de algunos países europeos y en Estados Unidos donde la educación, en especial la básica está orientada a desarrollar habilidades. Las habilidades se pueden entender como "La habilidad, en cualquiera de sus grados de desarrollo, se manifiesta en la ejecución del tipo de desempeños a los que dicha habilidad está referida; en otras palabras, las habilidades son constructos que se asocian a la realización de determinadas acciones que puede ejecutar el sujeto hábil; de allí que frecuentemente se utilicen de manera indistinta las expresiones "desarrollo 
de competencias" y "desarrollo de habilidades". ${ }^{1}$

La tendencia fue aceptada, pero no interiorizada y aquí viene el primer dilema en el que incurrió el MEN y fue promover unos procesos de evaluación por competencias, indicándole al educador que debía separarse de la evaluación de contenidos. Pero sin dejar en claro cuál era el cambio. Todo ellos afectado también por la formación de unos educadores bajo parámetros donde la educación era el desarrollo de contenidos para aprender.

Dentro del medio educativo y algunos profesores no diferencian la evaluación por competencias con el desarrollo de habilidades y a su vez estas con el desarrollo de habilidades de pensamiento. La solución que introdujo el Ministerio fue la creación de el PEI (programa educativo institucional) que debería construir cada institución educativa para fortalecer los planes de mejoramiento y trazarse unas metas de logro; sin embargo la mayoría de las instituciones educativas siguen basando su educación en la enseñanza de contenidos

\section{LA CONTRADICCIÓN: SEGUIR SATURANDO DE CONTENIDOS. ¿POR QUÉ PASA?}

Existe una contradicción en las instituciones educativas, porque exigen y promueven la evaluación por logros y competencias pero no han logrado desligarse de la impartición de contenidos, esto se evidencia especialmente en la escuelas - colegios privados. Los contenidos son importantes, teniendo en cuenta que el estudiante necesita unos pre conceptos y requiere de un elemento de agarre para desarrollar sus habilidades, eso requiere de una estructura básica y coherente de contenidos para desarrollar, pero algunos profesores confunden el desarrollo de habilidades con enseñar "lo que quieran" o "atiborrar de contenidos a los estudiantes".

Los hechos planteados atrás demuestran que el profesor sigue ordenando sus actividades académicas de enseñanza en la estructuración de contenidos y las áreas donde ha sido más difícil el cambio son ciencias naturales y sociales, justo dos áreas donde se empeña en saturar al estudiante de contenidos, exigen los mismos en los textos escolares y presionan con un supuesto argumento de la calidad en la necesidad de ofrecer la mayor cantidad de información posible. Por otra parte algunos profesores que intentan promover el desarrollo de habilidades y la evaluación por competencias, confunden el concepto de usar los contenidos como pretexto, y terminan por enseñar lo que está en sus gustos y no siempre eso está ligado a una estructura de conceptos básicos para que el estudiante se organice mentalmente.

\section{LA NECESIDAD DE GENERAR HÁBITOS DE LECTURA}

Un elemento que marca la diferencia en la educación de la mayoría de los países europeos, del sudeste asiático y Estados Unidos con la de América Latina y en este caso el Colombia, es la ausencia de hábitos del lectura. Los jóvenes europeos, del sudeste asiático y de Estados Unidos has desarrollado la habilidad de la lectura porque se ha implementado desde la primera infancia, los latinos no han desarrollado con la misma intensidad los hábitos de lectura; para los primeros la lectura se ha convertido en un hábito, es un placer...para los segundos una tarea, algo sólo compatible

1 Moreno Bayardo, Guadalupe. El desarrollo de habilidades como objetivo educativo. En: www.educacion.jalisco.gob. $\underline{\mathrm{mx}}$ 
en cuestiones de estudio. ¿Por qué no hemos logrado avances en la promoción de la lectura? ¿Dónde se presenta las debilidades?

Son los mismos procesos de formación que fallan, cuando se llena de contenidos a los estudiantes sin fundamento. En Estados Unidos, Japón, Reino Unido o Suecia se promueven los hábitos de lectura desde primera infancia y los estudiantes empiezan a leer y se convierte en un hábito, existen programas que incluyen el área de Ciencias Sociales donde se lee, se comparte la lectura, se hace en casa, en la escuela, además existe una cultura en la compra del libro, no es visto como un gasto, cómo un dolor de cabeza, como suelen hacerlo los medios de comunicación y las familias en el contexto colombiano...mientras tanto en la educación colombiana inducimos de contenidos, no se promueve el uso de lecturas para ampliar inquietudes, y el círculo vicioso hace que los profesores que fueron estudiantes dentro del sistema tampoco lean. La cuestión es que para desarrollar habilidad y hábito la lectura se convierte en una herramienta central, por eso en las universidad el principal medio de aprendizaje, para reflexionar, debatir y aprender, sigue siendo la lectura de textos y materiales previamente seleccionados por el educador.

Según los resultados de las pruebas Saber Pro 2011 (exámenes de Estado que se le aplican a los estudiantes de último semestre en la Universidad- anteriormente se llamaban Ecaes) el 25\% de los profesionales graduados no pueden producir un texto comprensible, ni organizado y en caso de los licenciados la cifra aumenta a 35\%. Ese nivel se presenta en el tercer año de secundaria en Estados Unidos, Francia o Reino Unido, según un estudio que presentó la revisa Semana. ${ }^{1}$

El círculo vicioso se mantiene, jóvenes que no disfrutan la lectura, se convierten en profesionales que no leen y que enseñan; no fomentan la lectura...no es una generalización, pero si, una tendencia muy marcada en la cultura educativa colombiana.

\section{¿SE COMPRENDE LA PROPUESTA DE ENSEÑAR POR COMPETENCIAS?}

En visitas realizadas y una encuesta aplicada en colegios de Barranquilla, Cali y Bogotá entre 2011 y 2012, se evidencia una tendencia que los profesores no comprenden el sentido de las competencias y las ligan al mero hecho de la evaluación y no como una estrategia de aprendizaje donde prime el desarrollo de habilidades. Existe un desconocimiento que se refleja en la priorización que se siguen dando a los contenidos y a los procesos de evaluación memorísticos en los colegios privados; por otra parte en los colegios públicos las actividades de las sociales se enfocan en la enseñanza de "vaguedades": valores, normas, emprendimiento y otras exigencias que plantea el Ministerios de Educación Nacional; y se abandonó por completo la disciplinariedad del área. Se percibe un desconocimiento generalizado de la enseñanza de la geografía y de la historia y a su vez del abordaje del desarrollo de habilidades. Por otra parte se aprecian niveles bajos de lectura en los educadores, lo que representa una problemática compleja como panorama general.

\section{EL CONFLICTO CON LOS TEXTOS ESCOLARES Y LOS PROCESOS DE FORMACIÓN DOCENTE}

En la experiencia en los procesos de elaboración y promoción de libros de textos se pueden evi- 
denciar dos marcadas posturas de los profesores frente al texto escolar. La primera la del profesor "tradicional" que quiere ver en el texto una enciclopedia, llena de contenidos, sin importar si esos temas son necesarios, valoran las enormes columnas de texto, cómo si existiera una relación directa entre número de palabras y párrafos, con la calidad del aprendizaje.

Por otra parte están los profesores que argumentan que los textos escolares los encasillan y que necesitan diferentes fuentes para generar habilidades en los estudiantes. Para ellos el texto escolar no es necesario y se plantean la elaboración de guías, talleres, que lamentable y contradictoriamente en un porcentaje muy alto están elaborados con "retazos de textos escolares", fotocopias, lecturas y demás complementos del libro de texto.

Cuando se trabaja el tema de las competencias y se explica que los contenidos son un medio para desarrollar habilidades, algunos educadores asumen que se puede enseñar con cualquier tema, que no se necesita un programa y que se enseña desde cualquier experiencia...y aunque existen temas particulares donde eso puede ser posible, debe existir un programa a desarrollar, es decir, deben existir unos contenidos y unas temáticas genéricas que permitan evaluar un proceso de enseñanzaaprendizaje, de lo contrario sin parámetros y políticas de seguimiento no existirán resultados.

Bajo esa premisa el libro de texto debe responder como una guía o mediador entre el profesor y el estudiante y debe responder a unas necesidades que orienten los procesos de aprendizaje con unas temáticas generales por nivel y grado de complejidad.

\section{UNA PROPUESTA HACIA DONDE DEBERÍAMOS IR}

Los textos escolares deber ser un guía de trabajo para el profesor y de aprendizaje para los estudiantes, deben ofrecer conocimientos y habilidades de trabajo que puedan aplicarse a la solución de problemas en la vida cotidiana. Para ello es necesario:

1. Secuenciar contenidos, es decir relacionar los programas de los cursos previos, para evidenciar una ilación y unas características de programa.

2. Presentar una claridad conceptual y expositiva con elementos gráficos, esquemas y actividades motivadoras. (cuando un estudiante es capaz de organizar la información en un esquema es porque se logró superar el aprendizaje memorístico.

3. Presentar una estricta secuenciación de contenidos, que evidencien niveles de complejidad e interpretaciones desde lo básico hasta lo complejo, desde los macro a lo micro o desde lo general a lo particular.

4. Potenciar la elaboración de esquemas mentales que faciliten la reflexión sobre lo que se hizo.

5. Ofrecer una amplia propuesta de actividades y ejercicios que faciliten un aprendizaje activo.

Con la anterior propuesta se estructuran los conocimientos previos, se motiva, hay una práctica secuenciada, se reflexiona lo que se hizo, se aplica a otras situaciones y se revisa lo que se hizo, es decir se desarrollan las habilidades en competencias básicas. 
Por lo tanto un libro de texto debe desarrollar un programa que evidencie fortalezas en varios aspectos cómo:

\section{La construcción de nuevos conocimientos donde se presente:}

- Una estricta secuenciación de contenidos que garanticen la exploración de conocimientos previos.

- La introducción de nuevos contenidos jerarquizados y ordenados de forma clara y concisa para facilitar el esquema-mental de los estudiantes.

- E1 desarrollo de ejercicios motivadores.

- La introducción de actividades didácticas para fomentar el aprendizaje y la adquisición de competencias básicas, lo que fortalece el aprendizaje activo.

El desarrollo de habilidades y capacidades por medio del tratamiento de información, al:

- Obtener información de fuentes diversas (fuentes históricas, mapas, imágenes, esquemas...).

- Presentar mapas conceptuales, esquemas... para organizar y comprender la información.

- Ofrecer imágenes y medios de comunicación de la información obtenida en diversos formatos.

- Aplicar los conocimientos obtenidos en contextos diferentes.

\section{Desarrollar habilidades específicas de las Ciencias Sociales con}

- El uso del tiempo y la cronología histórica.

- La ubicación en el espacio y el trabajo cartográfico.

- La comprensión de causas y consecuencias de los fenómenos sociales.

- La explicación en la continuidad y el cambio en los procesos históricos.

- La comprensión del lenguaje artístico.

- La valoración de la herencia cultural y del patrimonio histórico.

\section{Fortalecer otros tipo de competencias sociales con}

- El uso de las nuevas tecnologías de la información y la comunicación.

- Organizar y planificar el trabajo individual y en grupo. 
- Realizar pequeñas indagaciones que fomenten la búsqueda del conocimiento y la autonomía del aprendizaje.

- Desarrollar un pensamiento crítico y argumentativo.

- Ejercer la ciudadanía de forma responsable y democrática.

- Valorar el multiculturalismo social y desarrollar actitudes no-racistas, no-sexistas y tolerantes.

Los procesos de formación docente y el libro de texto

Para cerrar esta reflexión, es imposible lograr un cambio si no existe una conexión en las nuevas tendencias de formación y enseñanza en la escuela con los proceso de formación docente; porque la batuta de los cambios le corresponden a las nuevas generaciones de educadores que entran a las instituciones educativas cada año. Lo primero es hacer un verdadero análisis y reflexión sobre el papel de los textos escolares, ya que en la academia siempre ha existido críticas y prejuicios pre elaborados sobre el libro de texto.

En un artículo publicado en la revista Semana del 11 de marzo de 2012 aparece un documento donde un grupo de académicos pide al gobierno nacional y al ministerio de educación la separación de nuevo de la enseñanza de la geografía y de la historia, con un argumento válido, los jóvenes colombianos desconocen su pasado y por lo tanto les cuesta reflexionar el presente; de igual forma desconocen procesos espaciales y ambientales y las clases de sociales se han convertido en un collage de elementos desconectados. En esta crítica cuestionan los textos escolares por reforzar una historia romántica y tradicional y por ofrecer actividades memorísticas si ningún peso; sin embargo olvidan cinco de esos seis expertos que todos ellos han sido autores de libros de texto...entonces ¿los hacen por negocio?, ¿su discurso académico no se conecta con su posibilidad de elaborar textos escolares? La responsabilidad no es de un libro, se necesita interiorizar las necesidades en la educación y los libros responderán a esas necesidades; pero es imposible elaborar libros de texto con la propuesta arriba planteada cuando algunos educadores esperan los mismos esquemas tradicionales o no tienen claro el horizonte del tipo de formación que quieren impartir.

Allí de nuevo se evidencia la necesidad de promover hábitos de lectura en los futuros educadores, familiarizarlos con el libro de texto, desde diferentes perspectiva: la crítica, la revisión, la selección, las características, que se aprenda a valorar un texto de acuerdo a las necesidades de la educación, a los procesos de evaluación y se establezcan diferencias, porque en el universo del texto escolar como en la educación hay de todo y así como existen propuesta nuevas, hay otras que siguen imprimiendo el texto de hace veinte años con las mismas características y la lógica del mundo de dicha época.

\section{BIBLIOGRAFÍA}

Moreno Bayardo, G. (2012). El desarrollo de habilidades como objetivo educativo. Una aproximación Conceptual. En Revista Educar No6- Estrategias Comunicativas: 1998 (www.educacion.jalisco. gob.mx, visitada 13-5-2012) 